\title{
A rare case of right-sided varicocele in right renal tumor in the absence of venous thrombosis and IVC compression
}

\author{
Priyabrata Adhikari, Siddalingeshwar I. Neeli *i] and Shyam Mohan
}

\begin{abstract}
Background: The presence of unilateral right-sided varicocele hints at a serious retroperitoneal disease such as renal cell neoplasm. Such tumors are usually associated with a thrombus in renal vein or spermatic vein. We report a rare presentation of right-sided renal tumor causing right-sided varicocele in the absence of thrombus in renal vein and spermatic vein but due to an anomalous vein draining from the tumor into the spermatic vein as demonstrated by computed tomography angiogram.

Case presentation: A 54-yr-old hypertensive male presented with unilateral grade 3 right-sided varicocele and no other signs and symptoms. Ultrasound examination of his abdomen showed the presence of a mass lesion in the lower pole of right kidney. Computed tomography confirmed the presence of right renal mass, absence of thrombus in right renal vein or inferior vena cava. The angiographic phase of CT scan showed an anomalous vein from the tumor draining into the pampiniform plexus causing varicocele.

Conclusion: The presence of right-sided varicocele should raise a suspicion hidden serious pathological retroperitoneal condition, renal malignancy in particular, and should prompt the treating physician to carry out imaging studies of the retroperitoneum and careful study of the angiographic phase of the CT scan can ascertain the pathogenesis of the varicocele.
\end{abstract}

Keywords: Varicocele, Renal tumor, Retroperitoneal tumor

\section{Background}

The incidence of RCC has increased since 1970s by an average of $3-4 \%$ per year, largely related to the more prevalent use of USG and CT for the variety of presentations [1]. The common presentations of RCC aremacroscopic hematuria, flank pain, abdominal mass, lumbago, weight loss, malaise, paraneoplastic syndromes [2]. Varicocele is a rare presentation of RCC, especially on the right side [3]. The etiology of a right-sided varicocele includes impairment of the venous drainage of the pampiniform plexus by venous thrombosis, tumor invasion, or extrinsic compression by an intraabdominal

*Correspondence: sineeli@gmail.com

Department of Urology, Jawaharlal Nehru Medical College, KLES Dr

Prabhakar Kore Hospital and MRC, Nehrunagar, Belagavi 590010, India tumor [3]. We report a rare case of right-sided varicocele caused by right renal cell carcinoma tumor secondary to a vein draining the tumor into the right spermatic vein and not due to any compression or tumor thrombus in right renal vein, inferior vena cava or right testicular vein.

\section{Case presentation}

A 54-year old well controlled hypertensive male presented with painless, non-reducible swelling over right scrotum of one-month duration. He had no urinary complaints or fever. Physical examination of the patient revealed distended veins visible on the lateral aspect of right side of scrotum and thickened right cord with bag of worm feel on palpation which distended further on Valsalva maneuver suggestive of unilateral grade 3 right-sided varicocele (Fig. 1). Rest of 

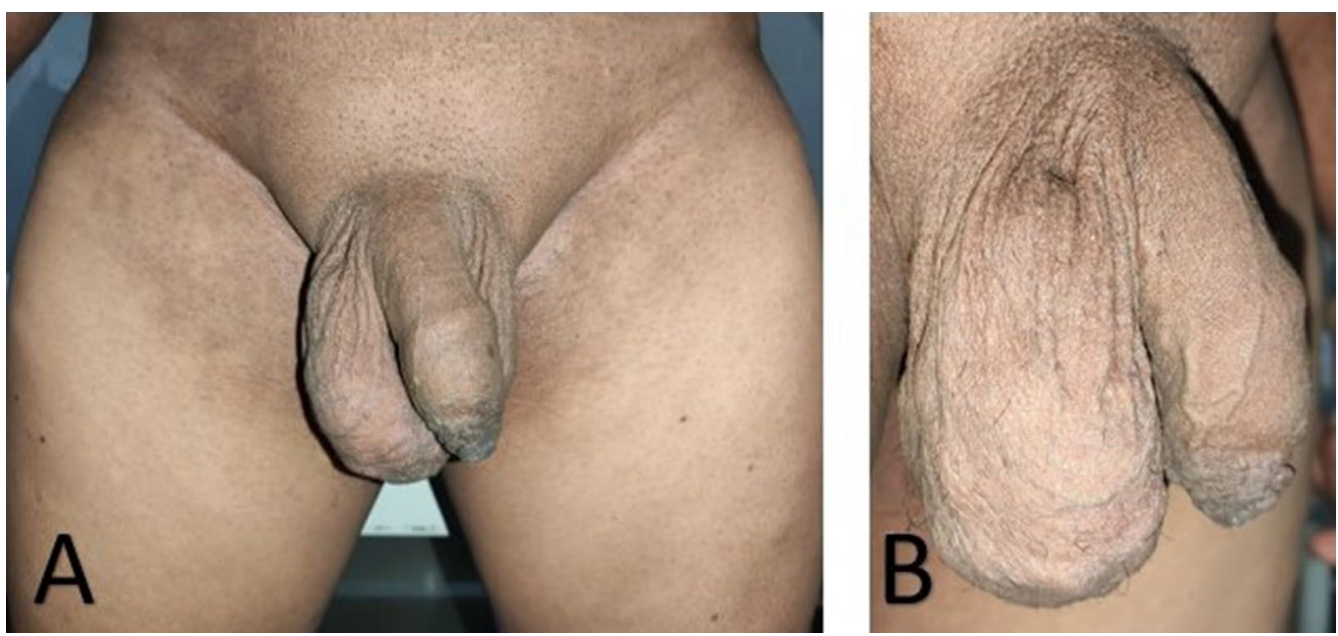

Fig. 1 a Unilateral grade 3 right varicocele; $\mathbf{b}$ Visible dilated veins over right scrotum

the clinical examination was within normal limits. His hemoglobin was $11 \mathrm{~g} / \mathrm{dL}$, total WBC count was 11,200 cells $/ \mathrm{mm}^{3}$ with $78 \%$ neutrophils (absolute neutrophil count of 8736 cells $/ \mathrm{mm}^{3}$ ), and platelet count was $4 \times 10^{5}$ cells $/ \mathrm{mm}^{3}$. He had serum creatinine of $1.1 \mathrm{mg} /$ $\mathrm{dL}$, serum calcium of $10.2 \mathrm{mg} / \mathrm{dL}$ and liver functions were within normal range. His urine examination was unremarkable. Ultrasound examination of the scrotum confirmed the presence of grade 3 varicocele and that of the abdomen showed a heterogeneous mass arising from the posterior aspect of lower pole of right kidney, suspicious of a malignant neoplasm. A contrast enhanced computed tomography (CECT) scan of the abdomen confirmed the presence of well-defined heterogeneously enhancing lower polar mass in the kidney measuring $6.7 \times 6.5 \times 5.4 \mathrm{~cm}$. No evidence of venous thrombosis or compression by tumor of the inferior vena cava, renal vein or spermatic vein was noted. No abdominal lymphadenopathy or intraabdominal metastases were found. The angiographic phase of the CT scan showed the tumor being hypervascular (Fig. 2) and an abnormal vein arising from the mass and draining into right spermatic vein, which was seen to be dilated and tortuous and resulting in the clinically evident right varicocele (Fig. 3). The diameter of the internal spermatic vein above the point of insertion of the anomalous vein was $3.8 \mathrm{~mm}$ and below the insertion it was $6.08 \mathrm{~mm}$., while the diameter of anomalous vein draining the tumor was $5 \mathrm{~mm}$. High-resolution CT (HRCT) of thorax showed randomly distributed nodular lesions in the superior segment of right upper lobe and lateral basal segment of left lower lobe suggestive of pulmonary metastases (Fig. 4). The biopsy from the

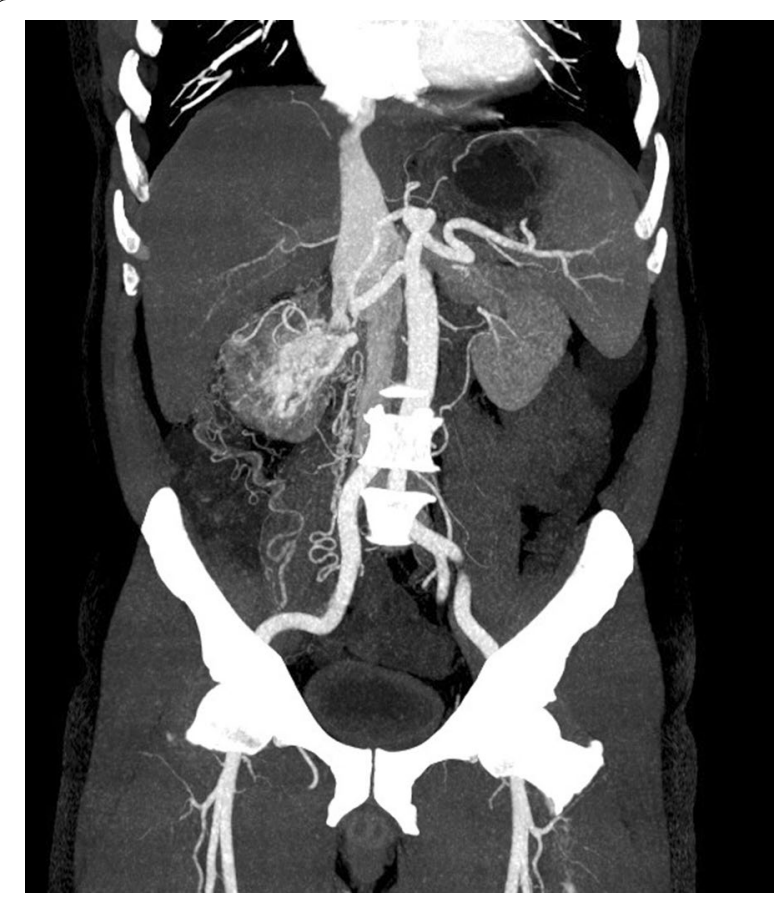

Fig. 2 Arteriographic phase showing leash of blood vessels supplying the tumor suggestive of hypervascularity/neovascularity of the tumor

renal mass suggested clear cell carcinoma (cT1bN0M1). In view of the presence of pulmonary metastases and the patient falling into intermediate risk category on IMDC risk model, he was started on sunitinib. After 6 months of follow-up, the patient continues to be asymptomatic and his right varicocele is persistent and has remained the same in size. 

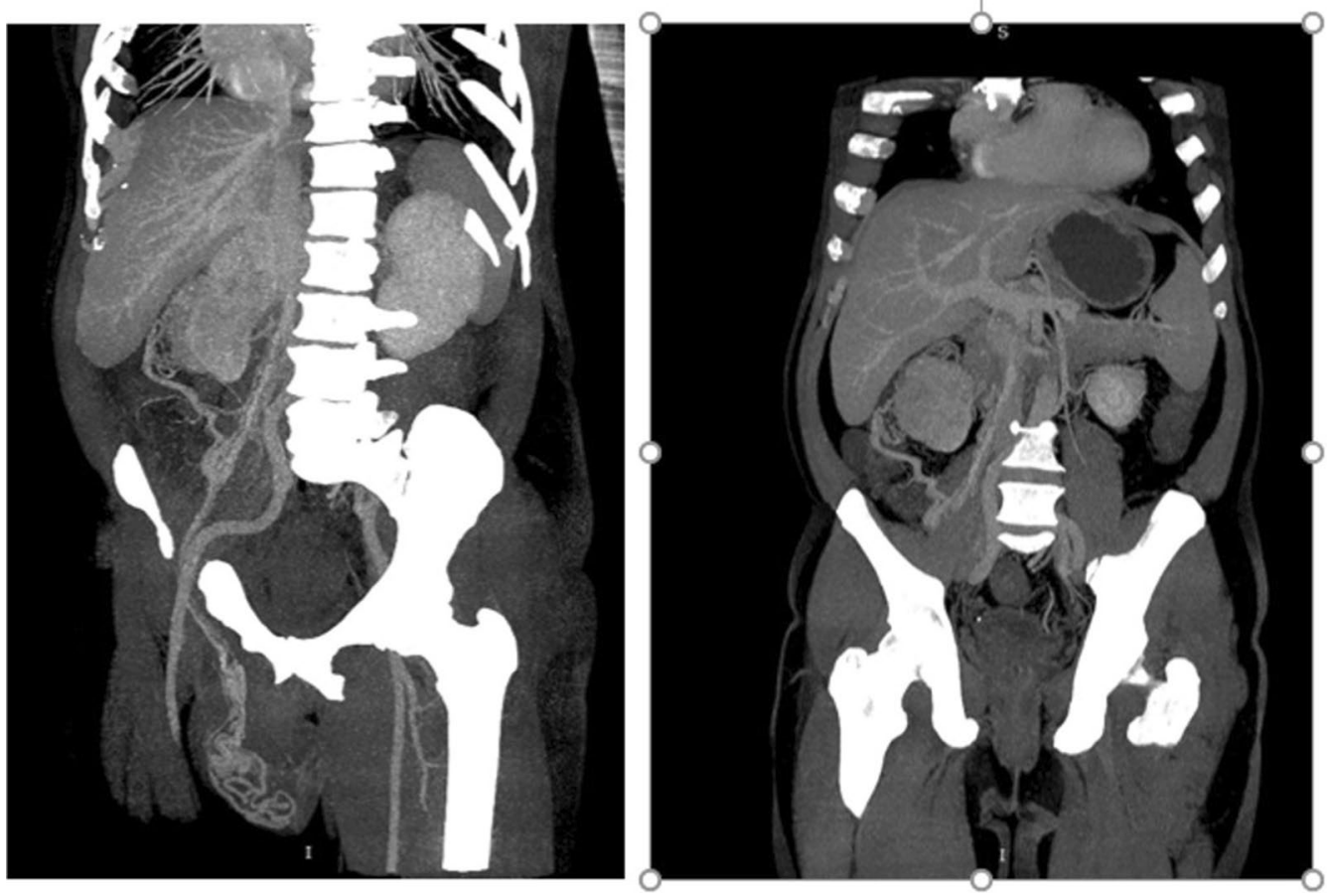

Fig. 3 Multiplanar reconstruction (MPR) images of CECT of abdomen showing an anomalous vein draining the right renal cell carcinoma into the right spermatic vein and resulting in right varicocele

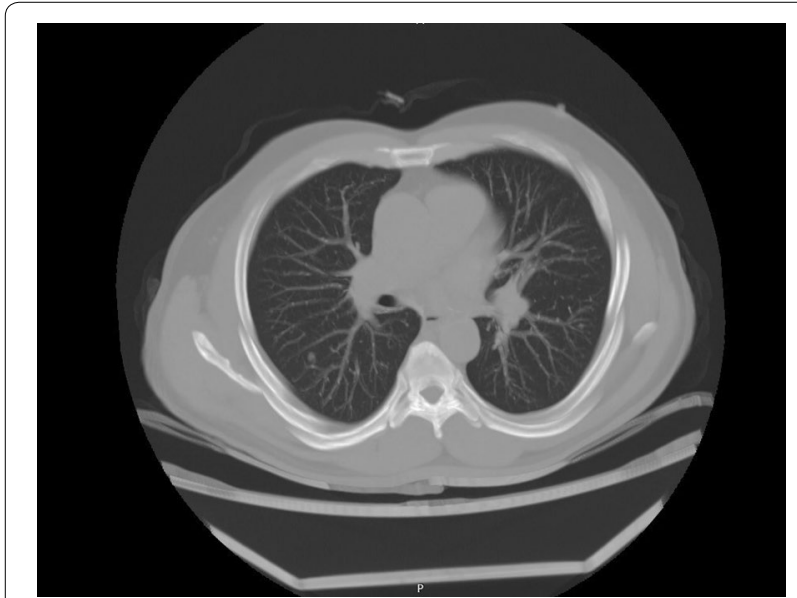

Fig. 4 HRCT of thorax showing nodular lesions suggestive of pulmonary metastasis

\section{Discussion}

Varicocele is defined as dilation of the pampiniform venous plexus draining the testicle [3]. Its diagnosis is primarily done by physical exam of the scrotum. Clinically the varicoceles are graded as follows: grade I varicocele (palpable only during Valsalva maneuver), grade II (palpable in the standing position), and grade III (visible without palpation). Among healthy men, the incidence of varicoceles varies from 8 to $22 \%$ [3]. Nearly 93\% varicoceles occur on the left side, while $25 \%$ are bilateral [4]. This occurs due to certain anatomical relationship between left renal vein and left testicular vein.

The etiology of varicoceles is multi-factorial. The common causes for left-sided varicocele include absent or incompetent venous valves, perpendicular insertion of internal spermatic vein into the left renal vein and nutcracker-like compression of the left renal vein between the aorta and superior mesenteric artery. On the other hand, the insertion of the right spermatic vein into the vena cava in an oblique orientation protects it from the high pressures of the vena cava. The right-sided varicoceles result from impairment of drainage of the spermatic vein either by a thrombus or compression $[3,4]$.

One clinical consequence of infrequency of solitary right varicocele is that should one be identified, retroperitoneal pathology such as renal tumor should be considered, especially if it is of abrupt onset [4]. While the reported incidence of varicocele in renal cell carcinoma varies from 0.6 to $11.4 \%$, the association of an isolated right varicocele and renal tumors is sparsely documented [5]. Various mechanisms have been described for the formation of right-sided varicocele in association with renal cell carcinoma such as extension of tumor thrombus into the spermatic vein with or without thrombus extending into inferior vena cava, invasion of right renal vein 
by the tumor causing dilatation of the gonadal vein and obstruction of inflow into duplicated testicular vein due to renal vein thrombus [6-9]. In the present case of right varicocele is caused by RCC without any involvement of tumor thrombus in IVC, right renal vein, right testicular vein and/or without any IVC compression by the tumor. In our report this patient has an anomalous vein draining the hypervascular right renal tumor to the right testicular vein causing dilatation and tortuosity as demonstrated by the CT angiogram. Parker et al. have reported a similar case where they used a varicocelogram to show increased flow from a venous tributary draining the tumor into the testicular vein resulting in right-sided varicocele [9]. Kulesza et al. [10] have reported an anatomical variation in which a venous tributary from the right pampiniform plexus drains into the right renal vein after traversing through the renal parenchyma and another tributary that drains into the right subcostal vein. They believed that these venous tributaries are remnants of a persistent subcardinal vein and an anastomosis of the supracardinal and subcardinal veins which was enmeshed into the growing metanephros. As these variant veins were found to have no valves, they postulated that these vessels may provide a venous channel for metastasis of renal cancer to the ipsilateral testis or for spread from testicular cancer to the ipsilateral testicle.

Parker et al. [9] documented reduction in the size of the varicocele after nephrectomy along with excision of the lateral venous tributary which was draining into the testicular vein. However, in the present case nephrectomy was not performed as the patient had pulmonary metastases at presentation. The hypervascularity of the tumor and the presence of anomalous veins may have caused hematogenous spread of the tumor resulting in pulmonary metastases in spite of the tumor being less than $7 \mathrm{~cm}$ (T1 tumor) and no obvious lymphadenopathy on the CT scan.

\section{Conclusion}

The present case reiterates the fact that despite poor understanding of pathophysiology of the occurrence of right-sided varicocele, its presence points toward a hidden serious pathological retroperitoneal condition such as renal neoplasm and should prompt the treating physician to carry out relevant imaging studies and careful study of the angiographic phase of the CT scan can ascertain the pathophysiology of the varicocele.

\section{Acknowledgements}

None.

\section{Authors' Contributions}

Study design was carried out by SN, PA; Data acquisition was carried out by PA SM, SN; Data analysis: was done by SN, PA; Drafting of manuscript was done by SN, PA, SM; Critical revision of the manuscript was carried out by SN. All authors have read and approved the manuscript.

\section{Funding}

Nil.

\section{Availability of data and materials}

Patient details available in the Medical Record Department of KLES Dr Prabhakar Kore Hospital and MRC, Belagavi.

\section{Ethics approval and consent to participate}

Not applicable.

\section{Consent for publication}

Written consent obtained from the patient (document written in kannada language, the vernacular language of the patient).

\section{Competing interests}

None.

Received: 21 May 2020 Accepted: 16 September 2020

Published online: 12 November 2020

\section{References}

1. Capitanio U, Bensalah K, Bex A et al (2019) Epidemiology of renal cell carcinoma. Eur Urol 75(1):74-84

2. Gibbons RP, Montie JE, Correa RJ et al (1976) Manifestations of renal cell carcinom. Urology 8(3):201-206

3. El-Saeity NS, Sidhu PS (2006) "Scrotal varicocele, exclude a renal tumour". Is this evidence based? Clin Radiol 61:593-599

4. Masson P, Brannigan RE (2014) The varicocele. Urol Clin North Am 41:129-144

5. Byrne GB, Hanna D, Townell N (1995) Right-sided varicocele as a presentation of right renal tumours. Br J Urol 75:798-799

6. Mabjeesh NJ, Bar-Yosef Y, Schreiber-Bramante L et al (2004) Spermatic vein tumor thrombus in renal cell carcinoma. Sci World J 4:192-194

7. Shinsaka H, Fujimoto N, Matsumoto T (2006) A rare case of right varicocele testis caused by a renal cell carcinoma thrombus in the spermatic vein. Int J Urol 13:844-845

8. Bonfitto M, Kimura LSM, Godoy JMP, Filho MZ et al (2019) Does rightsided varicocele indicate a right-sided kidney tumor? Archivio Italiano di Urologia e Andrologia 91(1):53-54

9. Parker HT (1975) Varicocele and renal tumor on right side. Urology 5(4):530-532

10. Kulesza R, Labranche L, Sweeney $S$ et al (2018) Identification of an aberrant testicular vein draining the right kidney. Int J Anat Var 11(1):15-17

\section{Publisher's Note}

Springer Nature remains neutral with regard to jurisdictional claims in published maps and institutional affiliations. 